\title{
Cervical Intramedullary Schwannoma with Syrinx: Case Report and Review of the Literature
}

\author{
Abdulaziz AlQarni ${ }^{1} \quad$ A. AlArifi ${ }^{1} \quad$ Ali H. Alassiri ${ }^{1} \quad$ Amjed Kouli ${ }^{1} \quad$ M. T. Abbas ${ }^{1}$ \\ ${ }^{1}$ Department of Neurosurgery, King Abdulaziz Medical City, \\ Saudi Arabia, Riyadh \\ Address for correspondence Abdulaziz AlQarni, MBBS, Department \\ of Surgery, King Abdulaziz Medical City, Ministry of National Guard, \\ P.O. Box: 22490, Riyadh 11426, Saudi Arabia, Riyadh \\ (e-mail: Dr.Abdulaziz_alqarni@yahoo.com).
}

\begin{abstract}
Keywords

- intramedullary schwannomas

- syrinx

- spinal cord tumor

- cervical

Schwannoma is a nerve sheath tumor originating from the Schwann cell. It is benign in nature and it arises from anywhere where Schwann cells can be found. It is rarely found in the parenchyma of the spinal cord. Intramedullary schwannomas (or neurilemmomas) without evidence of neurofibromatosis are rare spinal cord tumors. Intramedullary schwannoma was first reported in 1932 by Penfield. Our patient presented with neck pain, gradually worsening, weakness in the right upper and lower limbs, numbness in both shoulders, and a decrease in the grasping strength of both hands over a 4-year period. A magnetic resonance imaging of the spine showed a heterogeneously enhancing mass in the cervical spinal cord extending from the $\mathrm{C} 2$ to T1 levels with associated hemorrhagic changes. Histologically, the tumor was found to be composed of bland spindle cells with blunt-ended and sometimes wavy nuclei admixed with hyalinized vasculature. Surrounding reactive spinal cord parenchyma with frequent Rosenthal fibers was also observed. Focal Verocay bodies were evident, and with immunohistochemistry, there was diffuse and strong positivity for $\$ 100$, which is confirmatory for the diagnosis of schwannoma. We report a case of cervical intramedullary schwannoma presented with syringobulbia in a young adult.
\end{abstract}

\section{Introduction}

Schwannoma is a nerve sheath tumor originating from the Schwann cell. It is benign in nature and arises from anywhere where Schwann cells can be found. ${ }^{1}$ It is rarely found in the parenchyma of the spinal cord. ${ }^{2}$ Because Schwann cells do not exist in the central nervous system. Intramedullary schwannoma was first reported in 1932 by Penfield. ${ }^{3}$ One of the theories to explain the development of the tumor in this location is that it arises from the small bundles of peripheral nerves in the periphery of vasculature within the spinal cord. ${ }^{4,5}$ Other theorized origins are from anterior and posterior nerve roots that have extensions inside the spinal cord, or from metaplastic cells of the pia mater that may have differentiated into Schwann cells, or from neural crest cells that may have migrated to the spinal cord during fetal development. ${ }^{4,6}$ Most of the reported spinal cord schwannomas are found to be extramedullary. They have also been observed in the extradural space (25\%) and as a combination of intradural and extradural lesions (15\%); rarely have they been reported to be intramedullary. ${ }^{5,7}$ Intramedullary schwannoma accounts for almost 0.3 to $1.5 \%$ of all primary intraspinal lesions. ${ }^{8}$ It commonly involves the cervical region $(61 \%)$ and, to a lesser extent, the thoracic (29\%), and lumber (10\%) regions. ${ }^{1}$ Infrequently, they are associated with syringomyelia. ${ }^{4}$ To make a diagnosis of intramedullary schwannoma and to differentiate it from other neoplasms by imaging only is almost impossible. ${ }^{4}$ We can suspect an

\section{received}

July 31, 2016

accepted

October 18, 2016

published online

October 23, 2017
DOI https://doi.org/

10.1055/s-0037-1599788.

ISSN 2277-954X.

\author{
(C)2018 Neurological Surgeons' \\ Society of India
}

License terms

(®) $\Theta \circledast$ 
intramedullary schwannoma in patients with neurofibromatosis because approximately $20 \%$ of cases are associated with intramedullary lesions particularly schwannoma. ${ }^{1,9}$ We report a case of cervical intramedullary schwannoma occurring at the level between $\mathrm{C} 2$ and $\mathrm{T} 1$ and associated with syringobulbia.

\section{Case Report}

A 24-year-old male was admitted with complaints of neck pain, gradually worsening, weakness in the right upper and lower limbs, numbness in both shoulders, and a decrease in the grasping strength of both hands over a 4-year period. There was no history of urine or fecal incontinence. There was no history of trauma. He was not known to have any medical illness, and his family history was negative. On examination, he was well-built, conscious, and oriented. Glasgow Coma Scale was 15/15. The muscle power of the right upper and lower limbs was grade $4 / 5$ with spasticity and hyperreflexia. Both left upper and lower limbs had normal power, tone, and reflexes. The patient also displayed a hemiplegic gate. At the time of presentation, vibration, light touch, and position sense were all normal. A diagnosis of a cervical spinal cord lesion had been made at another institution. He was referred to our hospital for surgical management. A magnetic resonance imaging (MRI) at the spine showed a heterogeneously enhancing mass in the cervical spinal cord extending from the $\mathrm{C} 2$ to $\mathrm{T} 1$ levels with associated hemorrhagic changes ( $\mathbf{- F i g . ~ 1 A , ~ B ) . ~ T h e r e ~ w a s ~ a n ~ a s s o c i a t e d ~ s y r i n x ~ e x t e n d i n g ~}$ from the medulla oblongata to the lower thoracic cord. The appearance of the tumor was suggestive of an ependymoma. There were no specific brain findings. The patient was then prepared for surgery. The patient underwent awake endotracheal intubation and was given general anesthesia. He was placed prone and approached posteriorly through laminoplasty. Under neurophysiological monitoring, the dura was opened, and an exophytic part of the tumor was found at the level of C3, where the tumor was grossly totally resected (piece meal) using the microscope and Omni (dissection and suction). Postoperative MRI showed gross total removal of the tumor ( - Fig. 1C). In postoperative physical examination, the patient developed severe quadriparesis. Muscle power on the left side was $1 / 5$ and on the right was $3 / 5$. Upon histological examination, the tumor was found to be composed of bland spindle cells with blunt-ended and sometimes wavy nuclei admixed with hyalinized vasculature (-Fig. 2A-C). Surrounding reactive spinal cord parenchyma with frequent Rosenthal fibers was also observed. Focal Verocay bodies were evident, and with immunohistochemistry, there was diffuse and strong positivity for S100 (-Fig. 2D), which is confirmatory for the diagnosis of schwannoma.

\section{Discussion}

Schwannomas account for $30 \%$ of all intraspinal tumors, which are the commonest primary tumors of the spine. ${ }^{8}$ The age of patients ranges from 9 to 75 years (mean: 40.5 years). ${ }^{1}$ Intramedullary schwannoma is more frequently found in males than females (male:female $=3: 1$ ). ${ }^{10}$ The fourth decade of life is the mean age of onset of the symptoms. ${ }^{11}$ Pyramidal symptoms manifest most commonly and are followed by sensory disturbances and sphincter malfunction. This presentation is usually due to the slow compression of the spinal cord, which manifest as weakness, even though these tumors usually arise in the posterior portion of the spinal cord. ${ }^{7}$ In some cases, it has been reported that muscular fasciculations were the first clinical manifestation. ${ }^{10}$ The time between the beginning of the symptoms until diagnosis was almost always lengthy, with a mean of 28.2 months (range: 6 weeks to 12 years). ${ }^{7}$ Intramedullary schwannoma has three
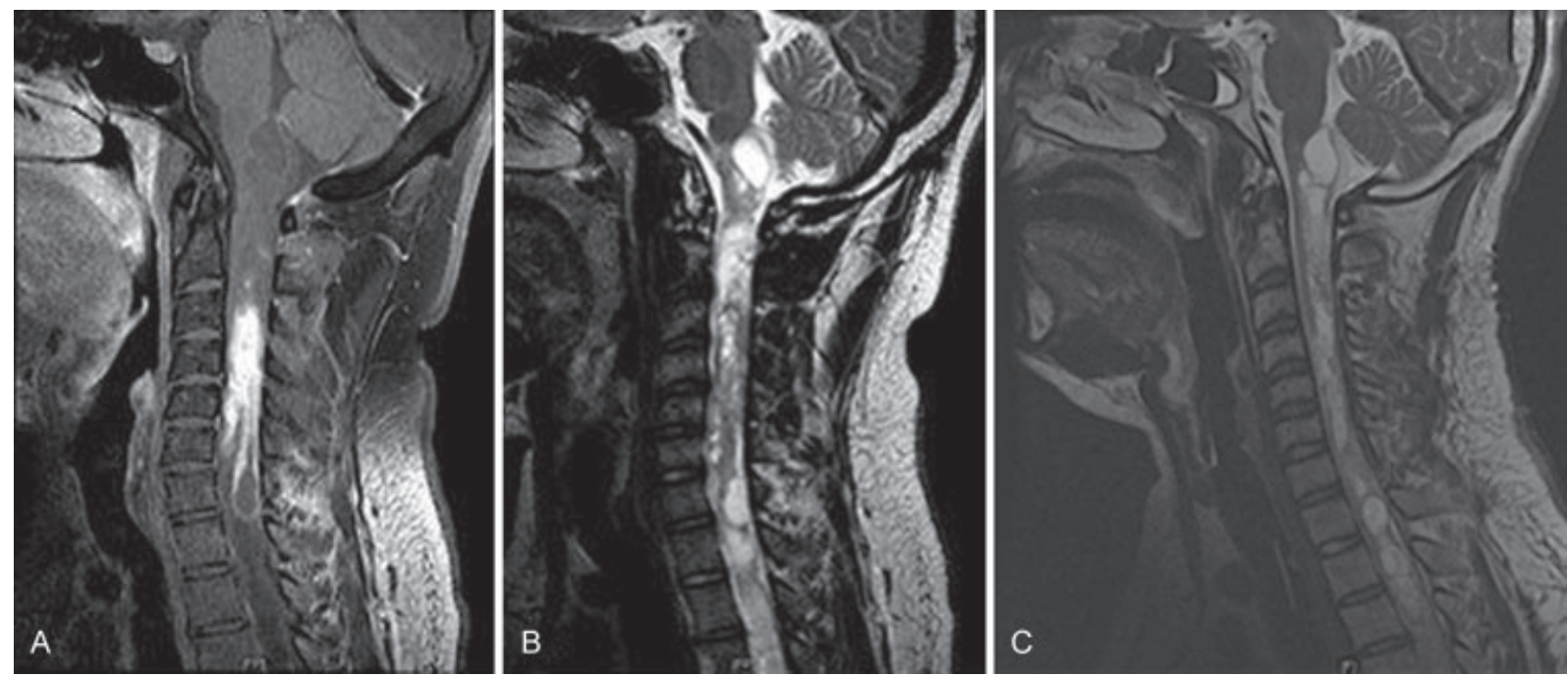

Fig. 1 (A) Contrast-enhanced T1-weighted magnetic resonance imaging (MRI) showing a heterogeneously enhanced mass lesion in the cervical spinal cord extending from C2 to T1 with syrinx extending from medulla oblongata to the lower thoracic cord. (B) T2-weighted MRI showing a heterogeneous hyperintense mass lesion in the cervical spinal cord extending from C2 to T1 with syrinx extending from medulla oblongata to the lower thoracic cord. (C) Postoperative T2-weighted MRI showing gross total removal of the tumor with regression of the syrinx. 

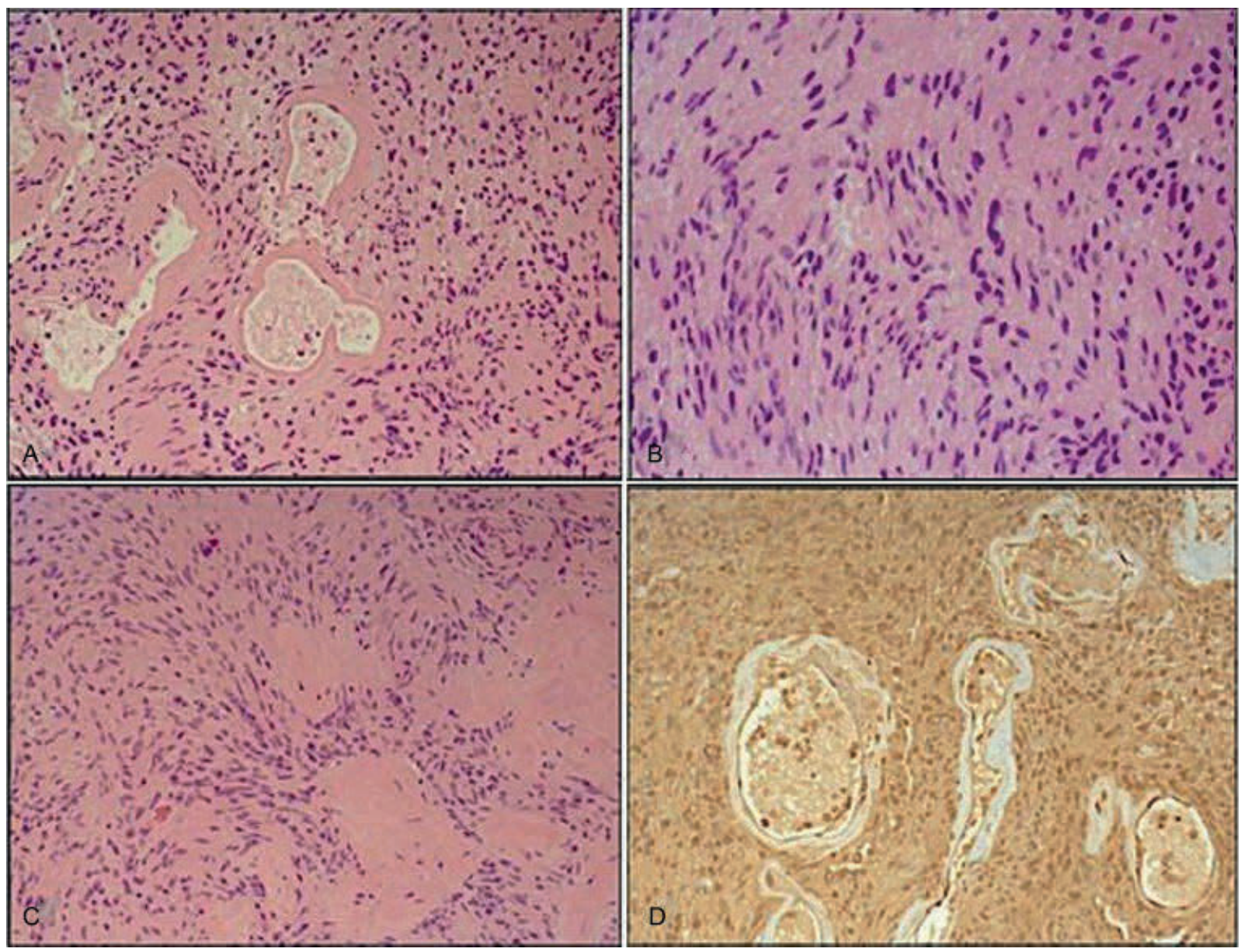

Fig. 2 (A) Benign appearing spindle cells and hyalinized vessels. (B) Tumor cells depicting blunt-ended and wavy nuclei. (C) Subtle palisading around nuclear free zones and more hyalinized vessels. (D) Positive immunoreactivity for S100 in nuclear and cytoplasmic patterns.

types based upon the lesion's location in the cross-sectional area of the spinal cord: central, surfacing, and dumbbell. ${ }^{12,13}$ The specific type may give a clue as to the origin of these tumors. In the central type, the lesion is found in the parenchyma, which supports the hypothesis that it originates from the perivascular nerve plexus or ectopic Schwann cells..$^{13}$ When the lesion is in the peripheral margin of the cord and attached to the pia mater, it named surfacing type, and this type suggests an origin from the Schwann cells of the posterior nerve roots or from conversion of pial cells., ${ }^{3,12,14,15}$ In only two cases of the surfacing type, it was suggested that the origin is from the anterior nerve root., ${ }^{4,13}$ Intra- and extramedullary schwannomas origin is considered to be from the dorsal root entry zone, which gives it the "dumbbell" shape. ${ }^{15}$ According to Kyoshima et al, eight cases of intra- and extramedullary schwannomas have been reported including their case..$^{13}$ Nearly $12 \%$ of intramedullary schwannoma patients are affected by neurofibroma. ${ }^{6}$ Cases of intramedullary schwannoma with neurofibromatosis that have been reported include five with neurofibromatosis type 1 and one with neurofibromatosis type $2 .{ }^{16,17}$ Also, Yang et al reported two cases with neurofibromatosis, and Lee et al reported one case but neither mentioned the type. ${ }^{18,19}$ Pediatric intramedullary schwannoma cases are rare.
To date, only seven pediatric intramedullary schwannomas have been reported in the literature. ${ }^{1,11,20-24}$ After reviewing these cases, we found that the age ranged from 8 to 15 years. All seven cases presented with sensory and motor deficits $(7 / 7 ; 100 \%)$. Pain was present in three $(42.8 \%)$ and genitourinary functional disturbance also 3 (42.8\%). Cervical lesions were found in four (57\%), thoracic in two (28.5), and one case involved the C6 to T1 levels. All seven cases underwent complete surgical resection, except for one who had a subtotal resection and an adjuvant radiotherapy. ${ }^{11}$ Partial or complete recovery was achieved in the majority of the cases postoperatively. To our knowledge, several authors did a literature review of intramedullary schwannomas since 1931. In 1986, Ross et al reviewed 25 cases, in 1991, Herregodts et al reviewed 36 cases, in 1999, Binatli et al, reviewed 57 cases, in 2002, Darwish et al reviewed 49 cases, and in 2005 , Kim et al found that a total of 69 cases had been reported. We have found that 48 cases were reported since 2005, which brings the total number of cases to 118 , including our case. ${ }^{1-3,8,10,11,13,18,19,23-37}$ The cases reported between 2005 and 2014 are summarized in - Tables $\mathbf{1}$ and $\mathbf{2}$. Lee et al studied 10 cases of intramedullary schwannomas that were diagnosed in their hospital from 1995 to 2010 and they found that 7 of them were in the lumber region and 3 in the cervical regions. 
Table 1 Summary of intramedullary schwannomas cases from 2005 to 2014

\begin{tabular}{|c|c|c|c|c|c|c|}
\hline Authors & $\begin{array}{l}\text { Age }(y) \text { and } \\
\text { sex }\end{array}$ & Location & Initial symptoms & $\begin{array}{l}\text { Duration of } \\
\text { symptoms }\end{array}$ & Treatment & Outcome \\
\hline Kim et al, 2005 & $72 \mathrm{M}$ & Thoracic T8-T9 & Left leg weakness and loss of sensation on the right side & $10 \mathrm{mo}$ & GTR & Improved \\
\hline Kyoshima et al, 2005 & $54 \mathrm{M}$ & Thoracic T9-10 & Numbness in the left foot and rectovesical dysfunction & $4 y$ & GTR & Improved \\
\hline Shenoy and Raja et al, 2005 & $29 M$ & Cervical C4-C7 & Interscapular pain that radiated to the upper limbs & $3 y$ & GTR & Improved \\
\hline Kahilogullari et al, 2005 & $34 \mathrm{~F}$ & $\begin{array}{l}\text { Thoracolumbar } \\
\text { T12-L2 "conus medullaris" }\end{array}$ & Pain around her waist and in her legs, and numbness & $7 \mathrm{mo}$ & STR & Improved \\
\hline Ho et al, 2006 & $45 \mathrm{M}$ & Cervical C5-C6 & Incidental & Incidental & GTR & Improved \\
\hline Ozawa et al, 2006 & $65 \mathrm{~F}$ & Cervical C2-C4 & Numbness of the left hand and paresthesia of the left leg & $2 y$ & GTR & Improved \\
\hline Mukerji et al, 2007 & $8 \mathrm{M}$ & Cervical C5-C7 & Weakness in all limbs & Sudden & GTR & Improved \\
\hline \multirow[t]{2}{*}{ Hida et al, 2008} & $41 \mathrm{M}$ & Cervical C1-C2 & Dysesthesia of all four limbs & $6 \mathrm{mo}$ & $\begin{array}{l}\text { Partial resection } \\
\text { then GTR }\end{array}$ & Improved \\
\hline & $30 \mathrm{M}$ & Cervical C5-C7 & Decrease in the grasping strength of the left hand & $?$ & $\begin{array}{l}\text { Partial resection } \\
\text { then GTR }\end{array}$ & Improved \\
\hline Hayashi et al, 2009 & $78 \mathrm{~F}$ & Thoracolumbar T11-L1 & Pain and numbness in both legs & $20 y$ & GTR & Improved \\
\hline Ohtonari et al, 2009 & $29 \mathrm{M}$ & $\begin{array}{l}\text { Thoracolumbar T12-L1 } \\
\text { "conus medullaris" }\end{array}$ & $\begin{array}{l}\text { Bladder dysfunction, sexual impotence, and } \\
\text { paresthesia in the buttocks }\end{array}$ & $8 \mathrm{mo}$ & STR & Improved \\
\hline Kim et al, 2009 & $11 \mathrm{~F}$ & Thoracic T5-T6 & $\begin{array}{l}\text { Weakness of the lower limbs, back pain, and urge } \\
\text { incontinence }\end{array}$ & $9 \mathrm{mo}$ & $S T R+R T$ & Improved \\
\hline Nicácio et al, 2009 & $40 \mathrm{M}$ & Cervical C4-C6 & Spastic tetraparesis and sphincterian disturbances & $2 y$ & STR & Improved \\
\hline Lyle et al, 2010 & Neonate & Thoracic T2 to the thecal sac & $?$ & $?$ & $?$ & $?$ \\
\hline Ryu et al, 2011 & $68 \mathrm{M}$ & Thoracic T5-T6 & Walking disturbance and decreased sensation & $17 \mathrm{mo}$ & GTR & Improved \\
\hline Vij et al, 2011 & $25 \mathrm{M}$ & Thoracic T10-T11 & $\begin{array}{l}\text { Low back pain radiating to right lower limb, and } \\
\text { bilateral weakness and numbness }\end{array}$ & $3 y$ & GTR & Improved \\
\hline Li et al, 2013 & $42 \mathrm{M}$ & Thoracic T3-T4 & $\begin{array}{l}\text { Zonesthesia in the right side of the chest, and } \\
\text { weakness and numbness of the bilateral lower limbs }\end{array}$ & $1.5 y$ & GTR & Improved \\
\hline Eljebbouri et al, 2013 & $10 \mathrm{M}$ & Thoracic T7-T9 & $\begin{array}{l}\text { Weakness of the lower limbs associated with bladder } \\
\text { and bowel incontinence }\end{array}$ & Sudden & GTR & Improved \\
\hline \multirow[t]{10}{*}{ Lee et al, 2013} & $19 \mathrm{~F}$ & Thoracic T6-T8 & \multirow{10}{*}{$\begin{array}{l}\text { Gait disturbance with motor deficit (nine cases) } \\
\text { associated with sensory disturbance (six cases,) } \\
\text { and difficulty in urination and toileting (two cases); } \\
\text { one patient presented with weakness in the left } \\
\text { upper limb }\end{array}$} & \multirow{10}{*}{$\begin{array}{l}\text { The mean } \\
\text { duration was } \\
39.3 \pm 36.0 \\
(\text { mo: } 3-120)\end{array}$} & GTR & Improved \\
\hline & $37 \mathrm{~F}$ & Thoracic T9-T10 & & & GTR & Improved \\
\hline & $39 \mathrm{~F}$ & Cervical C4-C7 & & & GTR & Improved \\
\hline & $41 \mathrm{~F}$ & Cervical C5-C6 & & & GTR & Improved \\
\hline & $42 \mathrm{M}$ & Thoracic T7-T8 & & & STR & Improved \\
\hline & $44 \mathrm{M}$ & Thoracic T8-T9 & & & GTR & Improved \\
\hline & $46 \mathrm{~F}$ & Thoracic T1-T2 & & & STR & Improved \\
\hline & $49 \mathrm{~F}$ & Cervical C5-C7 & & & STR & Improved \\
\hline & $60 \mathrm{M}$ & Thoracic T7-T10 & & & GTR & Improved \\
\hline & $78 \mathrm{M}$ & Thoracic T10-T11 & & & GTR & Improved \\
\hline
\end{tabular}


Table 1 (Continued)

\begin{tabular}{|c|c|c|c|c|c|c|}
\hline Authors & $\begin{array}{l}\text { Age }(y) \text { and } \\
\text { sex }\end{array}$ & Location & Initial symptoms & $\begin{array}{l}\text { Duration of } \\
\text { symptoms }\end{array}$ & Treatment & Outcome \\
\hline Karatay et al, 2014 & $30 \mathrm{~F}$ & $\begin{array}{l}\text { Thoracolumbar T12-L1 } \\
\text { "conus medullaris" }\end{array}$ & $\begin{array}{l}\text { Back pain, walking disturbance, and numbness in both } \\
\text { legs }\end{array}$ & $2 \mathrm{mo}$ & STR & Improved \\
\hline \multirow[t]{20}{*}{ Yang et al, 2014} & $17 \mathrm{M}$ & Thoracic T6-T8 & Right lower limb pain and numbness & $1 \mathrm{y}$ & STR & Improved \\
\hline & $31 \mathrm{M}$ & Cervical C3-C4 & Neck pain, bilateral upper limb numbness & $1 \mathrm{y}$ & GTR & Improved \\
\hline & $34 \mathrm{M}$ & Thoracic T12 & Back pain and left lower limb weakness & $4 y$ & GTR & Improved \\
\hline & $35 \mathrm{M}$ & Cervical C6 & Neck pain and left lower limb weakness & $3 y$ & GTR & Improved \\
\hline & $38 \mathrm{M}$ & Thoracic T11 & $\begin{array}{l}\text { Bilateral lower limb pain and numbness, and difficulty in } \\
\text { urination }\end{array}$ & $18 \mathrm{mo}$ & GTR & Improved \\
\hline & $39 \mathrm{M}$ & Cervical C3-C5 & Neck pain and bilateral lower limb weakness & $1 \mathrm{y}$ & GTR & Improved \\
\hline & $40 \mathrm{M}$ & Cervical C3 & Right upper limb pain and numbness & $2 \mathrm{mo}$ & GTR & Improved \\
\hline & $41 \mathrm{~F}$ & Cervical C4-C6 & Neck pain and bilateral lower limb weakness & $6 \mathrm{mo}$ & GTR & Improved \\
\hline & $42 \mathrm{M}$ & Thoracic T10- & Bilateral lower limb numbness & $2 y$ & GTR & Improved \\
\hline & $44 \mathrm{M}$ & T12 & Right lower limb numbness and weakness & $1 \mathrm{y}$ & GTR & Improved \\
\hline & $44 \mathrm{~F}$ & Thoracic T3 & $\begin{array}{l}\text { Thoracic and midback pain, and bilateral lower limb } \\
\text { weakness }\end{array}$ & $4 y$ & GTR & Improved \\
\hline & $46 \mathrm{M}$ & Cervical C5-C7 & $\begin{array}{l}\text { Back pain, and bilateral lower limb numbness and } \\
\text { weakness }\end{array}$ & $1 \mathrm{y}$ & STR & Improved \\
\hline & $48 \mathrm{M}$ & Thoracic T3-T5 & $\begin{array}{l}\text { Bilateral lower limb weakness, and numbness and difficul- } \\
\text { ty in urination }\end{array}$ & $12 \mathrm{y}$ & GTR & $\begin{array}{l}\text { No } \\
\text { change }\end{array}$ \\
\hline & $50 \mathrm{~F}$ & Thoracic T9-T10 & Neck and back pain, and right lower limb weakness & $2 y$ & GTR & Improved \\
\hline & $52 \mathrm{M}$ & Cervicothoracic C5-T1 & $\begin{array}{l}\text { Bilateral upper limb pain, bilateral lower limb weakness, } \\
\text { and difficulty in urination }\end{array}$ & $10 y$ & GTR & $\begin{array}{l}\text { No } \\
\text { change }\end{array}$ \\
\hline & $56 \mathrm{~F}$ & Cervicothoracic C6-T4 & $\begin{array}{l}\text { Neck pain, and bilateral lower limb numbness and } \\
\text { weakness }\end{array}$ & $3 y$ & STR & Improved \\
\hline & $57 \mathrm{M}$ & Cervical C5-C6 & Neck and back pain, and right upper limb pain & $6 \mathrm{mo}$ & GTR & Improved \\
\hline & $59 \mathrm{M}$ & Cervical C4-C6 & Right upper limb numbness and left lower limb pain & $3 y$ & STR & Improved \\
\hline & $60 \mathrm{~F}$ & Cervical C1-C2 & Bilateral lower limb pain and weakness & $3 y$ & GTR & Improved \\
\hline & $61 \mathrm{M}$ & $\begin{array}{l}\text { Thoracic T2-T3 } \\
\text { Cervical C6-C7 }\end{array}$ & Left upper limb pain and numbness & $2 y$ & GTR & Improved \\
\hline
\end{tabular}

Abbreviations: GTR, gross total resection; STR, subtotal resection.

Note: ? indicates that the value is not mentioned in the original paper. 
Table 2 Summary of MRI and histopathological findings in intramedullary schwannoma cases from 2005 to 2014

\begin{tabular}{|c|c|c|c|c|c|c|}
\hline Authors & T1-weighted image & T2-weighted image & $\begin{array}{l}\text { Gadolinium } \\
\text { enhancement }\end{array}$ & $\begin{array}{l}\text { Cysts, peritumoral } \\
\text { edema, or } \\
\text { syringomyelia }\end{array}$ & $\begin{array}{l}\text { Preoperative } \\
\text { diagnosis }\end{array}$ & $\begin{array}{l}\text { Histopathological } \\
\text { diagnosis }\end{array}$ \\
\hline Kim et al, 2005 & Isointense & Hyperintense & $\begin{array}{l}\text { Homogeneous; well } \\
\text { demarcated }\end{array}$ &,,-+- & $?$ & $\begin{array}{l}\text { Schwannoma, Antoni } \\
\text { type } A \text { and } B,+S 100 \\
\text { protein }\end{array}$ \\
\hline Kyoshima et al, 2005 & Hypo to isointense & Hypointense & $\begin{array}{l}\text { Homogeneous; well } \\
\text { demarcated }\end{array}$ &,,--- & $\begin{array}{l}\text { Intradural } \\
\text { extramedullary tumor }\end{array}$ & Schwannoma \\
\hline Shenoy and Raja, 2005 & Hypo to isointense & Hyperintense & $\begin{array}{l}\text { Ringlike peripheral } \\
\text { enhancement }\end{array}$ &,,--+ & $?$ & $\begin{array}{l}\text { Schwannoma, Antoni } \\
\text { type } A \text { and } B,+S 100 \\
\text { protein }\end{array}$ \\
\hline Kahilogullari et al, 2005 & $?$ & $?$ & Heterogeneous &,,--- & $?$ & $\begin{array}{l}\text { Schwannoma, Antoni } \\
\text { type } A,+S 100 \text { protein }\end{array}$ \\
\hline Ho et al, 2006 & Isointense & Hyperintense & $\begin{array}{l}\text { Homogeneous; well } \\
\text { demarcated }\end{array}$ &,,--- & Extramedullary tumor & $\begin{array}{l}\text { Schwannoma, Antoni } \\
\text { type A and B }\end{array}$ \\
\hline Ozawa et al, 2006 & Hypointense & Hyperintense & $\begin{array}{l}\text { Homogeneous; well } \\
\text { demarcated }\end{array}$ &,,-+- & Astrocytoma & $\begin{array}{l}\text { Schwannoma, Antoni } \\
\text { type A and B }\end{array}$ \\
\hline Mukerji et al, 2007 & $?$ & $?$ & $?$ &,,-+- & Astrocytoma & $?$ \\
\hline Hida et al, 2008 & $\begin{array}{l}\text { Hypointense } \\
\text { 2? }\end{array}$ & $\begin{array}{l}\text { Iso- to hyperintense } \\
?\end{array}$ & $\begin{array}{l}\text { Heterogeneous; well } \\
\text { demarcated } \\
\text { Homogeneous; well } \\
\text { demarcated }\end{array}$ & $\begin{array}{l}-,+,- \\
-,-,-\end{array}$ & $\begin{array}{l}\text { Schwannoma } \\
?\end{array}$ & $?$ \\
\hline Hayashi et al, 2009 & Hypointense & Isointense & Heterogeneous & $\begin{array}{l}-,-,- \\
\text { "calcification" }\end{array}$ & $?$ & $\begin{array}{l}\text { Ancient schwannoma, } \\
\text { Antoni type A, + S100 } \\
\text { protein + S100 } \\
\text { protein and GFAP }\end{array}$ \\
\hline Ohtonari et al, 2009 & Isointense & $?$ & $\begin{array}{l}\text { Homogeneous; well } \\
\text { demarcated }\end{array}$ &,,+-- & $?$ & $\begin{array}{l}\text { Schwannoma, Antoni } \\
\text { type } A,+\$ 100 \\
\text { protein }\end{array}$ \\
\hline Kim et al, 2009 & Hypointense & Hyperintense & $?$ &,,--+ & Ependymoma & $\begin{array}{l}\text { Schwannoma, Antoni } \\
\text { type } A \text { and } B,+S 100 \\
\text { protein }\end{array}$ \\
\hline Nicácio et al, 2009 & Hypointense & Hyperintense & $\begin{array}{l}\text { Heterogeneous; well } \\
\text { demarcated }\end{array}$ &,,--+ & $?$ & $\begin{array}{l}\text { Schwannoma, Antoni } \\
\text { type A }\end{array}$ \\
\hline Lyle et al, 2010 & $?$ & $?$ & $?$ & $?$ & $?$ & $\begin{array}{l}\text { Congenital } \\
\text { schwannoma, Antoni } \\
\text { type A and B. }\end{array}$ \\
\hline
\end{tabular}


Table 2 (Continued)

\begin{tabular}{|c|c|c|c|c|c|c|}
\hline Authors & T1-weighted image & T2-weighted image & $\begin{array}{l}\text { Gadolinium } \\
\text { enhancement }\end{array}$ & $\begin{array}{l}\text { Cysts, peritumoral } \\
\text { edema, or } \\
\text { syringomyelia }\end{array}$ & $\begin{array}{l}\text { Preoperative } \\
\text { diagnosis }\end{array}$ & $\begin{array}{l}\text { Histopathological } \\
\text { diagnosis }\end{array}$ \\
\hline Ryu et al, 2011 & Hypointense & Hyperintense & $\begin{array}{l}\text { Heterogeneous; well } \\
\text { demarcated }\end{array}$ &,,-+- & $?$ & $\begin{array}{l}\text { Schwannoma, Antoni } \\
\text { type } A,+S 100 \\
\text { protein and silver stain }\end{array}$ \\
\hline Vij et al, 2011 & Hypointense & Isointense & $\begin{array}{l}\text { Homogeneous; well } \\
\text { demarcated }\end{array}$ &,,--- & Ependymoma & $\begin{array}{l}\text { Schwannoma, Antoni } \\
\text { type A, with } \\
\text { cysticercus parasite cyst }\end{array}$ \\
\hline Li et al, 2013 & Isointense & Hypointense & $\begin{array}{l}\text { Heterogeneous; well } \\
\text { demarcated }\end{array}$ &,,--- & $\begin{array}{l}\text { Astrocytoma or } \\
\text { ependymoma }\end{array}$ & Schwannoma? \\
\hline Eljebbouri et al, 2013 & $?$ & Hyperintense & Heterogeneous &,,+--- & Astrocytoma & $\begin{array}{l}\text { Schwannoma, Antoni } \\
\text { type A and B. }\end{array}$ \\
\hline Lee et al, 2013 & $\begin{array}{l}\text { Hyperintense in three } \\
\text { cases; hypointense in } \\
\text { four cases; and iso- } \\
\text { intense in three cases }\end{array}$ & $\begin{array}{l}\text { Hyperintense in five } \\
\text { cases and hypointense } \\
\text { in five cases }\end{array}$ & $\begin{array}{l}\text { Six cases with a } \\
\text { homogenous, well- } \\
\text { enhanced mass with } \\
\text { sharp demarcation, } \\
\text { two cases with a } \\
\text { heterogeneous } \\
\text { enhanced mass, and } \\
\text { two cases with } \\
\text { peripheral enhancement }\end{array}$ & $\begin{array}{l}\text { Peritumoral edema } \\
\text { (seven cases,) and } \\
\text { tumor cysts } \\
\text { (eight cases) }\end{array}$ & $\begin{array}{l}\text { Ependymomas in four } \\
\text { cases, astrocytomas } \\
\text { in three cases, and } \\
\text { hemangioblastoma, } \\
\text { lymphoma, and } \\
\text { metastasis in one } \\
\text { case each }\end{array}$ & $\begin{array}{l}\text { All Schwannomas, Antoni } \\
\text { type } A \text { and } B \\
+ \text { S100 protein }\end{array}$ \\
\hline Karatay et al, 2014 & Hypointense & Hyperintense & $\begin{array}{l}\text { Homogeneous; well } \\
\text { demarcated }\end{array}$ &,,--+ & $?$ & $\begin{array}{l}\text { Schwannoma, Antoni } \\
\text { type } A \text { and } B,+S 100 \text { protein }\end{array}$ \\
\hline \multirow[t]{8}{*}{ Yang et al, 2014} & Isointense & Isointense & $\begin{array}{l}\text { Heterogeneous; well } \\
\text { demarcated }\end{array}$ &,,-+- & Astrocytoma & $\begin{array}{l}\text { All schwannomas, } \\
\text { Antoni type A and B }\end{array}$ \\
\hline & Isointense & Isointense & $\begin{array}{l}\text { Heterogeneous; well } \\
\text { demarcated }\end{array}$ &,,--- & Ependymoma & \\
\hline & Isointense & Iso- to hyperintense & $\begin{array}{l}\text { Heterogeneous; well } \\
\text { demarcated }\end{array}$ &,,+-+ & Schwannoma & \\
\hline & Hyperintense & Hyperintense & $\begin{array}{l}\text { Homogeneous; well } \\
\text { demarcated }\end{array}$ &,,+-+ & Ependymoma & \\
\hline & Isointense & Isointense & $\begin{array}{l}\text { Homogeneous; well } \\
\text { demarcated }\end{array}$ &,,--+ & Ependymoma & \\
\hline & Isointense & Isointense & $\begin{array}{l}\text { Homogeneous; well } \\
\text { demarcated }\end{array}$ &,,--- & Ependymoma & \\
\hline & Isointense & Hyperintense & $\begin{array}{l}\text { Heterogeneous; well } \\
\text { demarcated }\end{array}$ &,,--+ & Ependymoma & \\
\hline & Hyperintense & Hyperintense & $\begin{array}{l}\text { Homogeneous; well } \\
\text { demarcated }\end{array}$ &,,--+ & Ependymoma & \\
\hline
\end{tabular}


Table 2 (Continued)

\begin{tabular}{|c|c|c|c|c|c|c|}
\hline Authors & T1-weighted image & T2-weighted image & $\begin{array}{l}\text { Gadolinium } \\
\text { enhancement }\end{array}$ & $\begin{array}{l}\text { Cysts, peritumoral } \\
\text { edema, or } \\
\text { syringomyelia }\end{array}$ & $\begin{array}{l}\text { Preoperative } \\
\text { diagnosis }\end{array}$ & $\begin{array}{l}\text { Histopathologica } \\
\text { diagnosis }\end{array}$ \\
\hline & Iso- to hypointense & Iso- to hyperintense & $\begin{array}{l}\text { Heterogeneous; well } \\
\text { demarcated }\end{array}$ &,,--+ & Ependymoma & \\
\hline & Isointense & Isointense & $\begin{array}{l}\text { Homogeneous; well } \\
\text { demarcated }\end{array}$ &,,+-- & Schwannoma & \\
\hline & Isointense & Iso- to hyperintense & $\begin{array}{l}\text { Heterogeneous; well } \\
\text { demarcated }\end{array}$ &,,-+- & Ependymoma & \\
\hline & Iso- to hypointense & Hyperintense & Heterogeneous &,,++- & Ependymoma & \\
\hline & Isointense & Hyperintense & $\begin{array}{l}\text { Homogeneous; well } \\
\text { demarcated }\end{array}$ &,,--+ & Astrocytoma & \\
\hline & Hypointense & Iso- to hyperintense & $\begin{array}{l}\text { Homogeneous; well } \\
\text { demarcated }\end{array}$ &,,++- & Ependymoma & \\
\hline & Iso- to hypointense & Hyperintense & $\begin{array}{l}\text { Heterogeneous; well } \\
\text { demarcated }\end{array}$ &,,++- & Ependymoma & \\
\hline & Hypointense & Hyperintense & Heterogeneous &,,++- & Ependymoma & \\
\hline & Iso- to hypointense & Hyperintense & $\begin{array}{l}\text { Heterogeneous; well } \\
\text { demarcated }\end{array}$ &,,++- & Astrocytoma & \\
\hline & Isointense & Isointense & $\begin{array}{l}\text { Homogeneous; well } \\
\text { demarcated }\end{array}$ &,,--+ & Ependymoma & \\
\hline & Isointense & Isointense & Heterogeneous &,,--+ & Ependymoma & \\
\hline & Hypointense & Iso- to hyperintense & $\begin{array}{l}\text { Circular; well } \\
\text { demarcated }\end{array}$ &,,+-- & Ependymoma & \\
\hline
\end{tabular}

Abbreviations: GFAP, glial fibrillary acidic protein; MRI, magnetic resonance imaging.

Note: ? indicates that the value is not mentioned in the original paper.

+ indicates that the value exists in the images

- indicates that the value does not exist in the images 
The primary radiological images suggested the diagnosis of ependymoma in four cases, astrocytoma in three cases, and hemangioblastoma, lymphoma, and metastasis in the other three cases. The T1-weighted MRI images revealed the lesion with hyperintensity in three cases, hypointensity in four cases, and an isointensity in three cases. The T2-weighted MRI images revealed the lesion with hyperintensity in five cases and with hypointensity in five cases. The T1-weighted MRI with contrast resulted in homogenous, well-enhanced tumors which were well-demarcated in six cases, a heterogeneous enhanced tumor in two cases, and peripheral enhancement in two cases. Lesions were accompanied by perilesional edema in seven cases and cysts in eight cases. Of 365 patients with a diagnosis of spinal cord schwannomas included in the study, only 10 (2.7\%) had intramedullary schwannomas. The first symptom was gait abnormality with motor deficit, followed by sensory deficit and urinary symptoms. Histologically, all tumors showed Antoni A and B areas, and mitotic figures were hardly found. In the immunohistochemical tests, all tumor cells were positive for S100 protein but negative for glial fibrillary acidic protein. This study supports the theory of the nerve root origin of intramedullary schwannoma because half of the tumors had an attachment to the dorsal rootlets. ${ }^{18} \mathrm{Wu}$ et al reviewed the data of seven patients with diagnosed intramedullary schwannoma treated at their hospital from 2003 to 2010 and compared them with patients with ependymoma and astrocytoma from the same period. They found that there was a significant difference in the incidence of somatic and root pain as the first symptoms between intramedullary schwannoma and ependymoma $(p=0.005)$ and between intramedullary schwannoma and astrocytoma $(p=0.019)$, but not between ependymoma and astrocytoma $(p=0.175)$. MRI analysis showed isointense or low-intense tumors on T1-weighted images and high intense or mixed on T2-weighted images. There were four cases associated with tumor cysts and three cases with syringomyelia above the tumor. Contrast enhancement was homogenous in four cases, heterogeneous in two, and circular in one. All lesions were well-demarcated. But they did not find a significant difference on MRI between intramedullary schwannoma and the gliomas. The mean postoperative follow-up period was 56 months, with no neurologic deterioration or recurrence. ${ }^{38}$ Yang et al analyzed 20 cases of intramedullary schwannoma that were diagnosed in their hospital from 2000 to 2013, including 7 cases that were reported by Wu et al in 2011, but with a longer follow-up period ( - Table 1). They also encountered 1,320 patients with intramedullary lesions (ependymomas, astrocytomas, and hemangioblastomas) and 1,723 patients with intraspinal schwannomas during the same period. Intramedullary schwannomas accounted for $1.49 \%$ of all intramedullary lesions (total $=1,320$ ) and $1.16 \%$ of all intraspinal schwannomas (total $=1,723) .{ }^{19}$ Intramedullary schwannomas are commonly found on MRI T1-weighted images as iso- or hypointense lesions, and a little hyperintense on T2-weighted images. ${ }^{7}$ The lesions are most commonly well marginated, with some edema. ${ }^{7}$ With contrast enhancement, almost all cases show homogeneous or nodular enhanced tumors. ${ }^{7}$ Ependymomas, astrocytomas, hemangioblastomas, and metastasis are all intramedullary lesions that are contrast-enhanced. Therefore, they are included in the differential diagnosis. ${ }^{7,39}$ Commonly, they show vague lesion margins and are accompanied by edema and cysts. ${ }^{40-42}$ On the contrary, intramedullary schwannomas usually reveal a greatly enhanced lesion with well-demarcated margins. ${ }^{4,7}$ Most ependymomas are centrally located because they grow from the central canal, with a symmetrical expansion of the spinal cord. ${ }^{41}$ Astrocytomas originate from the astrocyte, which can be found anywhere in the spinal cord, and therefore their growth patterns tend to be different. ${ }^{41}$ Hemangioblastomas have a prominent enhancement and may have a flow void on MRI. ${ }^{42} \mathrm{~A}$ review of 20 cases by Ozawa et al concluded that 10 cases showed hypointensity to isointensity signals on T1-weighted MRI and slightly hyperintensity signals in 5 cases. T2-weighted MRI revealed seven cases with hyperintensity signals and five cases with hypointensity signals. Contrast-enhanced T1-weighted MRI resulted in well-defined lesion margins in 15 cases. Homogenous enhancement was documented in $65 \%$ of cases. Perilesional edema was mentioned in 10 cases. ${ }^{30}$ Balériaux concluded that there is no correlation between the MRI findings and the Antoni classification. ${ }^{39}$ The Antoni A type is characterized by the presence of condensed wavy bundles of cells with rod or ovoid nuclei, and palisading arrangement of the cells can be seen. ${ }^{1}$ The Antoni B type has large and loosely organized hypodense cells with polymorphism. ${ }^{1}$ The new WHO classification of tumors has three types of schwannomas: plexiform, cellular, and melanotic. ${ }^{11}$ Complete excision of intramedullary schwannoma is usually achievable, and postoperative outcomes have been good..$^{28}$ However, there were reports of death in five cases and recurrence in two. ${ }^{1,43}$ Ohtonari et al analyzed the resectability of 39 cases of intramedullary schwannomas of the spinal cord at each level and found 5 subtotal excision and 17 total excision cases at the cervical level, 4 and 6 at the thoracic level, 2 and 5 at the lumber level. All cases with total excision showed no neurologic deterioration. Two cases worsened after subtotal excision. One was at the cervical level and the other was at the lumber level. ${ }^{2}$ Recurrence after subtotal excision of intramedullary schwannomas was reported in two cases after 5 and 3 years follow-up. Even though the final histological diagnosis of the two cases were schwannomas, subtotal removal was done because frozen sections during the operation suggested astrocytoma. ${ }^{43}$

\section{Conclusion}

Intramedullary schwannomas are benign and slowly progressive lesions. The definitive diagnosis can be made by pathology. It is difficult to differentiate intramedullary schwannoma from other intramedullary lesions by MRI only. When gross total resection is usually needed but cannot be done, subtotal resection of the tumor is recommended. A good clinical outcome after surgery can be anticipated. 


\section{References}

1 Binatli O, Erşahin Y, Korkmaz O, Bayol U. Intramedullary schwannoma of the spinal cord. A case report and review of the literature. J Neurosurg Sci 1999;43(02):163-167, discussion 167-168

2 Ohtonari T, Nishihara N, Ota T, Ota S, Koyama T. Intramedullary schwannoma of the conus medullaris complicated by dense adhesion to neural tissue. Neurol Med Chir (Tokyo) 2009;49(11):536-538

3 Ross DA, Edwards MS, Wilson CB. Intramedullary neurilemomas of the spinal cord: report of two cases and review of the literature. Neurosurgery 1986;19(03):458-464

4 Kodama Y, Terae S, Hida K, Chu BC, Kaneko K, Miyasaka K. Intramedullary schwannoma of the spinal cord: report of two cases. Neuroradiology 2001;43(07):567-571

5 Colosimo C, Cerase A, Denaro L, Maira G, Greco R. Magnetic resonance imaging of intramedullary spinal cord schwannomas. Report of two cases and review of the literature. J Neurosurg 2003;99(1, Suppl):114-117

6 Conti P, Pansini G, Mouchaty H, Capuano C, Conti R. Spinal neurinomas: retrospective analysis and long-term outcome of 179 consecutively operated cases and review of the literature. Surg Neurol 2004;61(01):34-43, discussion 44

7 Riffaud L, Morandi X, Massengo S, Carsin-Nicol B, Heresbach N, Guegan Y. MRI of intramedullary spinal schwannomas: case report and review of the literature. Neuroradiology 2000;42(04):275-279

8 Ho T, Tai KS, Fan YW, Leong LL. Intramedullary spinal schwannoma: case report and review of preoperative magnetic resonance imaging features. Asian J Surg 2006;29(04):306-308

9 Nadkarni TD, Rekate HL. Pediatric intramedullary spinal cord tumors. Critical review of the literature. Childs Nerv Syst 1999;15(01):17-28

10 Nicácio JM, Rodrigues JC, Galles MH, Faquini IV, de Brito Pereira CA, Ganau M. Cervical intramedullary schwannoma: a case report and review of the literature. Rare Tumors 2009;1(02):e44

11 Kim NR, Suh YL, Shin HJ. Thoracic pediatric intramedullary schwannoma: report of a case. Pediatr Neurosurg 2009;45(05):396-401

12 Cantore G, Ciappetta P, Delfini R, Vagnozzi R, Nolletti A. Intramedullary spinal neurinomas. Report of two cases. J Neurosurg 1982;57(01):143-147

13 Kyoshima K, Horiuchi T, Zenisaka H, Nakazato F. Thoracic dumbbell intra- and extramedullary schwannoma. J Clin Neurosci 2005;12(04):481-484

14 Cambier J, Masson M, Hurth M, Poirier J, Dehen H. Unilateral posterior-column syndrome due to intramedullary neurinoma. Imitative homolateral synkinesias [in French] Rev Neurol (Paris) 1974;130(5-6):189-199

15 Wood WG, Rothman LM, Nussbaum BE. Intramedullary neurilemoma of the cervical spinal cord. Case report. J Neurosurg 1975;42(04):465-468

16 Morimoto T, Sasaki T, Mochizuki T, Takakura K, Sato A. Thoracic intramedullary neurinoma with multiple intracranial meningiomas; case report [in Japanese] No Shinkei Geka 1992;20(04):423-427

17 Bhayani R, Goel A. Multiple intramedullary schwannomascase report. Neurol Med Chir (Tokyo) 1996;36(07):466-468

18 Lee SE, Chung CK, Kim HJ. Intramedullary schwannomas: long-term outcomes of ten operated cases. J Neurooncol 2013;113(01):75-81

19 Yang T, Wu L, Deng X, Yang C, Xu Y. Clinical features and surgical outcomes of intramedullary schwannomas. Acta Neurochir (Wien) 2014;156(09):1789-1797

20 Rasmussen TB, Kernohan JW, Adson AW. Pathologic classification, with surgical consideration, of intraspinal tumors. Ann Surg 1940;111(04):513-530
21 Gorman PH, Rigamonti D, Joslyn JN. Intramedullary and extramedullary schwannoma of the cervical spinal cord-case report. Surg Neurol 1989;32(06):459-462

22 Sharma SC, Ray RC, Banerjee AK. Intramedullary spinal schwannoma. Indian Pediatr 1989;26(03):290-292

23 Mukerji G, Sherekar S, Yadav YR, Chandrakar SK, Raina VK. Pediatric intramedullary schwannoma without neurofibromatosis. Neurol India 2007;55(01):54-56

24 Eljebbouri B, Gazzaz M, Akhaddar A, Elmostarchid B, Boucetta M. Pediatric intramedullary schwannoma without neurofibromatosis: case report. Acta Med Iran 2013;51(10):727-729

25 Herregodts P, Vloeberghs M, Schmedding E, Goossens A, Stadnik T, D'Haens J. Solitary dorsal intramedullary schwannoma. Case report. J Neurosurg 1991;74(05):816-820

26 Darwish BS, Balakrishnan V, Maitra R. Intramedullary ancient schwannoma of the cervical spinal cord: case report and review of literature. J Clin Neurosci 2002;9(03):321-323

27 Kahilogullari G, Aydin Z, Ayten M, Attar A, Erdem A. Schwannoma of the conus medullaris. J Clin Neurosci 2005;12(01):80-81

28 Kim SD, Nakagawa H, Mizuno J, Inoue T. Thoracic subpial intramedullary schwannoma involving a ventral nerve root: a case report and review of the literature. Surg Neurol 2005;63(04):389-393, discussion 393

29 Shenoy SN, Raja A. Cystic cervical intramedullary schwannoma with syringomyelia. Neurol India 2005;53(02):224-225

30 Ozawa N, Tashiro T, Okamura T, Koyama K, Ohata K, Inoue Y. Subpial schwannoma of the cervical spinal cord mimicking an intramedullary tumor. Radiat Med 2006;24(10):690-694

31 Hida K, Yano S, Iwasaki Y. Staged operation for huge cervical intramedullary schwannoma: report of two cases. Neurosurgery 2008;62(05, Suppl 2):ONS456-ONS460, discussion ONS460

32 Hayashi F, Sakai T, Sairyo K, et al. Intramedullary schwannoma with calcification of the epiconus. Spine J 2009;9(05):e19-e23

33 Lyle CA, Malicki D, Senac MO, Levy ML, Crawford JR. Congenital giant intramedullary spinal cord schwannoma. Neurology 2010;75(19):1752

34 Ryu KS, Lee KY, Lee HJ, Park CK. Thoracic intramedullary schwannoma accompanying by extramedullary beads-like daughter schwanommas. J Korean Neurosurg Soc 2011;49(05):302-304

35 Vij M, Jaiswal S, Jaiswal AK, Behari S. Coexisting intramedullary schwannoma with intramedullary cysticercus: report of an unusual collision. Indian J Pathol Microbiol 2011;54(04):866-867

36 Li J, Ke Y, Huang M, Li Z, Wu Y. Microexcision of intramedullary schwannoma at the thoracic vertebra. Exp Ther Med 2013;5(03):845-847

37 Karatay M, Koktekir E, Erdem Y, Celik H, Sertbas I, Bayar MA. Intramedullary schwannoma of conus medullaris with syringomyelia. Asian J Surg 2014 (e-pub ahead of print). Doi: 10.1016/j.asjsur.2014.04.004

38 Wu L, Yao N, Chen D, Deng X, Xu Y. Preoperative diagnosis of intramedullary spinal schwannomas. Neurol Med Chir (Tokyo) 2011;51(09):630-634

39 Balériaux DL. Spinal cord tumors. Eur Radiol 1999;9(07): $1252-1258$

40 Nemoto Y, Inoue Y, Tashiro T, et al. Intramedullary spinal cord tumors: significance of associated hemorrhage at MR imaging. Radiology 1992;182(03):793-796

41 Miyazawa N, Hida K, Iwasaki Y, Koyanagi I, Abe H. MRI at $1.5 \mathrm{~T}$ of intramedullary ependymoma and classification of pattern of contrast enhancement. Neuroradiology 2000;42(11):828-832

42 Chu BC, Terae S, Hida K, Furukawa M, Abe S, Miyasaka K. MR findings in spinal hemangioblastoma: correlation with symptoms and with angiographic and surgical findings. AJNR Am J Neuroradiol 2001;22(01):206-217

43 Duong H, Tampieri D, Melançon D, Salazar A, Robert F, Alwatban J. Intramedullary schwannoma. Can Assoc Radiol J 1995;46(03):179-182 